\section{Loss of the cylindromatosis} tumour suppressor inhibits apoptosis by activating NF- $\mathrm{\kappa}$

\section{Thijn R. Brummelkamp ${ }^{\star}$, Sebastian M. B. Nijman*, Annette M. G. Dirac ${ }^{\star}$ \& René Bernards}

Division of Molecular Carcinogenesis and Center for Biomedical Genetics, The Netherlands Cancer Institute, Plesmanlaan 121, 1066 CX Amsterdam, The Netherlands

* These authors contributed equally to this work

Protein modification by the conjugation of ubiquitin moietiesubiquitination-plays a major part in many biological processes, including cell cycle and apoptosis ${ }^{1}$. The enzymes that mediate ubiquitin-conjugation have been well-studied, but much less is known about the ubiquitin-specific proteases that mediate deubiquitination of cellular substrates ${ }^{2,3}$. To study this gene family, we designed a collection of RNA interference vectors to suppress 50 human de-ubiquitinating enzymes, and used these vectors to identify de-ubiquitinating enzymes in cancer-relevant pathways. We report here that inhibition of one of these enzymes, the familial cylindromatosis tumour suppressor gene $(C Y L D)^{4}$, having no known function, enhances activation of the transcription factor NF-кB. We show that CYLD binds to the NEMO (also known as IKK $\gamma$ ) component of the IKB kinase (IKK) complex, and appears to regulate its activity through de-ubiquitination of TRAF2, as TRAF2 ubiquitination can be modulated by CYLD. Inhibition of CYLD increases resistance to apoptosis, suggesting a mechanism through which loss of CYLD contributes to oncogenesis. We show that this effect can be relieved by aspirin derivatives that inhibit NF- $\mathrm{kB}$ activity ${ }^{5}$, which suggests a therapeutic intervention strategy to restore growth control in patients suffering from familial cylindromatosis.

The family of de-ubiquitinating enzymes (DUBs) consists of ubiquitin carboxy-terminal hydrolases and ubiquitin-specific processing proteases ${ }^{1-3}$. A role for DUB genes in cancer is suggested by the fact that this family contains both oncogenes ${ }^{6,7}$ and tumour suppressor genes ${ }^{4}$. The strategy we pursued to study the function of the family of DUB enzymes was to inhibit expression of individual family members through RNA interference and search for phenotypes. A total of 50 genes containing the conserved catalytic domain of DUB enzymes could be identified in sequence databases (Fig. 1a, and Supplementary Information). We retrieved the complementary DNA sequences, and selected four unique 19-mer sequences from each transcript for cloning into PSUPER, a vector that mediates suppression of gene expression through synthesis of short hairpin RNAs having small interfering RNA (siRNA)-like properties ${ }^{8}$. We chose to make four knockdown vectors against each DUB to increase the chance that a significant inhibition of DUB expression would be obtained. In total, we made 200 knockdown vectors, which were subsequently pooled into 50 sets of 4 vectors, where each set of vectors was designed to target a single DUB transcript (Fig. 1a).

To ask how effective sets of four knockdown vectors inhibited DUB gene expression, we fused the open reading frame of four of the DUBs to green fluorescent protein (GFP) and determined the levels of GFP-DUB fusion protein expression in the absence and presence of co-expression of the DUB knockdown vectors. A significant reduction in protein levels was induced by all four DUB knockdown vector pools, whereas control p21-red fluorescent protein (RFP) fusion protein was unaffected (Fig. 1b). We conclude that this strategy allows efficient inhibition of DUB expression.

To study members of the DUB family, we asked if suppression of
DUBs affected the activity of NF- $\mathrm{B}$, a transcription factor involved in inflammation, immune responses and protection against apoptosis ${ }^{9}$. We transfected an NF-kB-luciferase reporter gene, together with each of the 50 sets of 4 DUB knockdown vectors into human U2-OS cells, and measured the effect of DUB knockdown on tumour necrosis factor- $\alpha$ (TNF- $\alpha)$-activation of NF-кB. Only one of the sets of DUB knockdown vectors (no. 36) enhanced TNF- $\alpha$-activation of NF-кB (Fig. 1c). Importantly, the DUB no. 36 set of knockdown vectors targets the cylindromatosis tumour suppressor gene $C Y L D^{4}$ (see Supplementary Information). Levels of haemagglutinin (HA)-CYLD protein levels were significantly reduced by the most active of the four knockdown vectors (pSUPER-CYLD) (Fig. 1d). CYLD knockdown did not enhance basal level of NF-кB activity, but caused a further increase in NF-кB activation by phorbol 12-myristate 13-acetate (PMA) (Fig. 1e). This indicates that CYLD loss activates NF- $\mathrm{KB}$ in response to diverse stimuli. Together, these data suggest that CYLD is an $\mathrm{NF}-\mathrm{\kappa} \mathrm{B}$ regulator.

NF- $\kappa$ B is inactivated by IкB proteins. Signalling through the IкB kinase (IKK) complex, containing the IкB kinases IKK $\alpha$ and $\beta$ and the structural component NEMO (or IKK $\gamma$ ), causes phosphorylation and subsequent degradation of IкB, allowing activation of NF-кB ${ }^{9,10}$. The observed effect of CYLD on NF-кB activity could result from an effect of CYLD on the TNF- $\alpha$ receptor, the TNFreceptor-associated factor (TRAF) proteins, a downstream effect on the IKK complex or on the IкB/NF-кB complex. To address this, we asked if CYLD associates with members of the NF- $\kappa B$ signalling machinery. We found that CYLD co-immunoprecipitated with NEMO (Fig. 2a). Since CYLD interacts with NEMO in a yeast two hybrid assay (see accompanying manuscript ${ }^{11}$ ), this is likely to be direct. However, owing to the lower expression of Iк $\mathrm{B} \alpha$ and IKK $\beta$ (Fig. 2a), we cannot exclude the possibility that CYLD also interacts with other components of the IKK complex.

To study the effect of CYLD on IKK activity, we measured IKK $\beta$ activity in an in vitro kinase assay. In the absence of TNF- $\alpha$ no IKK $\beta$ kinase activity towards IкB $\alpha$ could be detected (Fig. 2b). As expected, TNF- $\alpha$ treatment stimulated IKK $\beta$ kinase activity. Importantly, this activity was enhanced when cells were co-transfected with pSUPER-CYLD (compare Fig. 2b, lanes 5, 6). No effects were seen of CYLD knockdown on IKK $\beta$ protein levels (Fig. 2b, lower panel), suggesting that CYLD does not regulate IKK $\beta$ abundance. Consistent with increased IKK $\beta$ kinase activity by pSUPER-CYLD, we observed that CYLD knockdown resulted in a reduction in IкB $\alpha$ levels, an endogenous substrate of IKK $\beta$ kinase (Fig. 2c). This decrease in IкB $\alpha$ level occurs without TNF- $\alpha$ treatment, indicating that there might be some IKK activity in U2-OS cells under the tissue culture conditions used, or that CYLD is required to maintain shut-off of IKK signalling in the absence of activating signals. These data indicate that CYLD acts as an antagonist of the IKK complex that binds to the NEMO component, and that a reduction in CYLD stimulates IKK-activity. A prediction from these data is that expression of a catalytically inactive mutant of CYLD will also cause a reduction of the IкB $\alpha$ levels through competition with endogenous CYLD. Figure $2 \mathrm{~d}$ shows that transfection of cells with a point mutant of CYLD in which the active site cysteine is mutated ${ }^{7}$ causes a similar reduction in $\mathrm{I} \kappa \mathrm{B} \alpha$ levels as seen with pSUPERCYLD (compare Fig. 2d, lanes 2 and 4). We conclude that the de-ubiquitinating enzyme activity of CYLD is required to act on NF-кB.

TNF receptor-associated factors (TRAFs) are involved in cytokine-mediated activation of NF- $\mathrm{B}^{12,13}$. NF-кB-inducing activity of TRAF2 and TRAF6 requires their ubiquitin ligase activity and non-destructive lysine-63 poly-ubiquitination ${ }^{14,15}$. Their ubiquitination during TNF signalling makes these proteins candidate substrates for CYLD. We co-transfected TRAF2 and HA-ubiquitin expression vectors, and monitored levels of TRAF2 ubiquitination in the presence of both wild-type CYLD and an active site mutant 
of CYLD. Figure 2e shows that TRAF2 is ubiquitinated, in agreement with the notion that TRAF2 over-expression leads to activation of $\mathrm{NF}-\mathrm{KB}^{11}$. Importantly, co-expression of wild-type CYLD caused a reduction in TRAF2 ubiquitination, and an active site mutant of CYLD markedly increased levels of ubiquitinated TRAF2 (Fig. 2e). Although these results do not rule out the possibility that CYLD could have other targets in the pathway, or that the effects seen on TRAF2 ubiquitination are indirect, they are consistent with a model in which the effect of CYLD on NF-кB activation is mediated by CYLD-dependent de-ubiquitination of TRAF2.

When combined with inhibitors of transcription or translation, TNF- $\alpha$ can be a potent inducer of apoptosis ${ }^{16-19}$. This pro-apoptotic activity of TNF- $\alpha$ can be inhibited by activation of NF- $\kappa$ B, which activates a number of anti-apoptotic genes ${ }^{20}$. Since CYLD knockdown stimulates PMA-induced activation of NF- $\mathrm{BB}$, we asked if CYLD and PMA also collaborate to inhibit TNF- $\alpha$-induced apoptosis. HeLa cells were treated with TNF- $\alpha$ in the presence of cycloheximide (CHX) to induce apoptosis both with and without pre-treatment with PMA, which stimulates expression of antiapoptotic NF-кB target genes (see Methods). Figure 3a, b shows that treatment with TNF- $\alpha$ efficiently induced apoptosis in some $95 \%$ of the HeLa cells, as judged by TUNEL assay (data not shown). As expected, pre-treatment with PMA resulted in an approximately four-fold increase of the number of viable cells. Significantly, PMA pre-treatment in CYLD knockdown cells a

Selected DUB genes
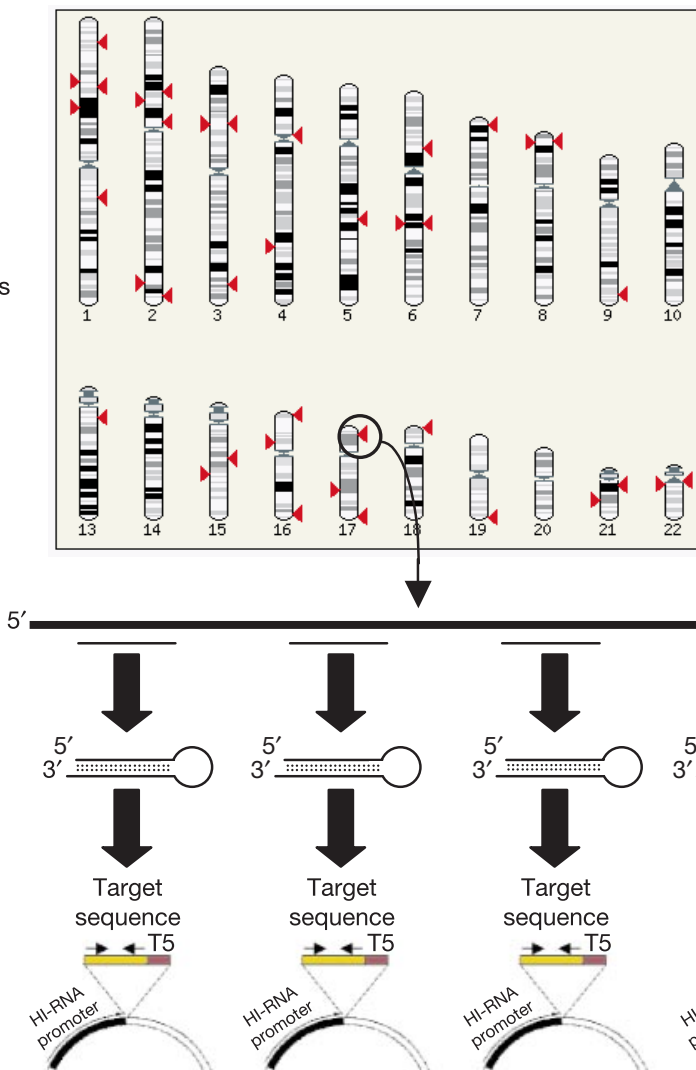

pSUPER

Cloning in PSUPER
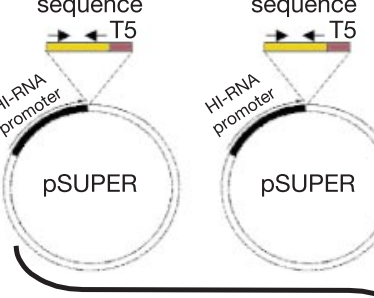

SSUPER
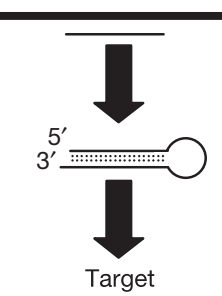

sequence

$\rightarrow \leftarrow$ T5

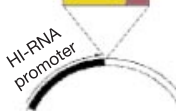

pSUPER

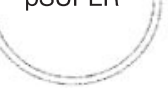

DUB knock

b

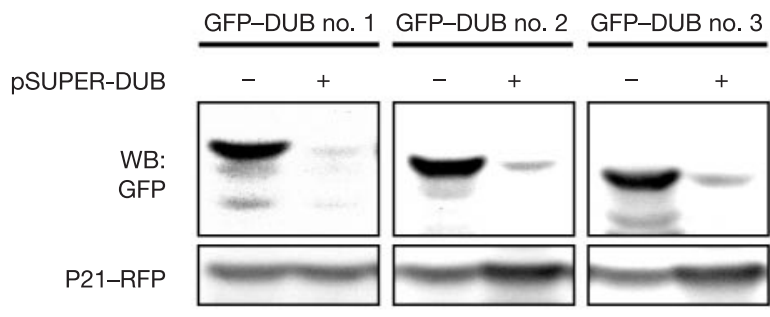

Figure 1 Genome-wide DUB knockdown screen. a, Overview of identified DUBs using the Ensembl database, and construction of the knockdown library. b. HEK293 cells were transfected and immunoblotted against GFP or p21-RFP (control). WB, western blot. c, U2-OS cells were co-transfected with a NF-кB luciferase reporter and individual members of the DUB knockdown library. Cells were stimulated with TNF- $\alpha$ and luciferase

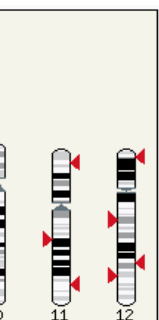

c

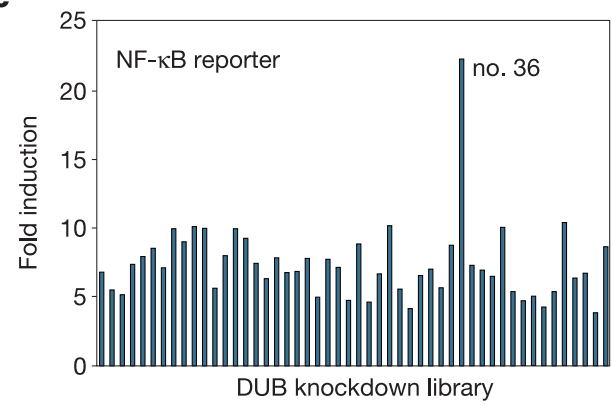

d
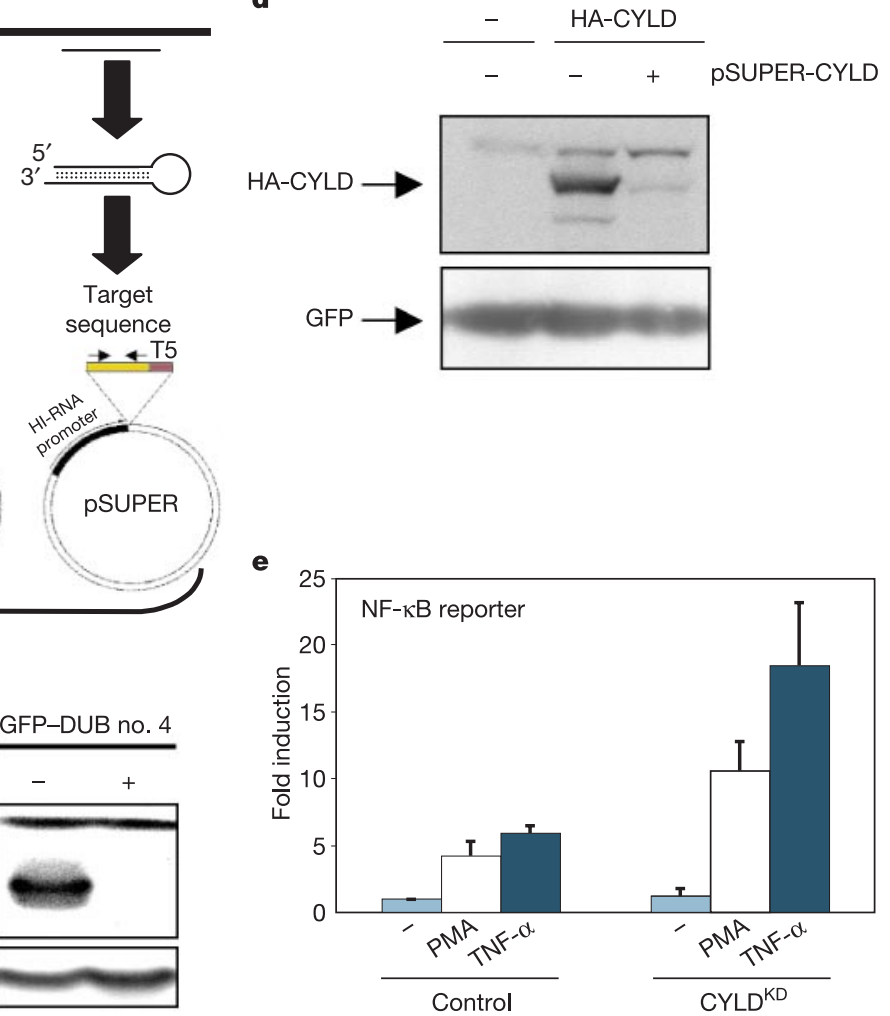
resulted in an even larger fraction of surviving cells, suggesting that CYLD loss can be anti-apoptotic (Fig. 3a, b), probably through enhanced activation of NF-кB. Consistent with this, CYLD knockdown and PMA also collaborated in NF-кB activation in HeLa cells (Fig. 3c).

NF-кB can be inhibited by a number of pharmacological agents, including sodium salicylate (an aspirin derivative) and prostaglandin A1 (PGA1) $)^{5,21}$. Both inhibit IKK $\beta$, the kinase which is hyper-activated as a result of CYLD knockdown (Fig. 2b). To address whether the effect of CYLD knockdown on NF-кB activation can be counteracted by sodium salicylate or PGA1, we transfected U2-OS cells with the NF-кB-reporter and stimulated NF-кB with PMA. As observed before, pSUPER-CYLD enhanced PMA-stimulated NF-кB activity (Fig. 4a, b). This effect of CYLD knockdown on NF-кB could be suppressed by both sodium salicylate and PGA1 (Fig. 4a, b), indicating that these compounds can compensate for CYLD suppression in this assay.

It is likely that loss of CYLD confers resistance to apoptosis through activation of NF-кB. If correct, the protective effect of

a

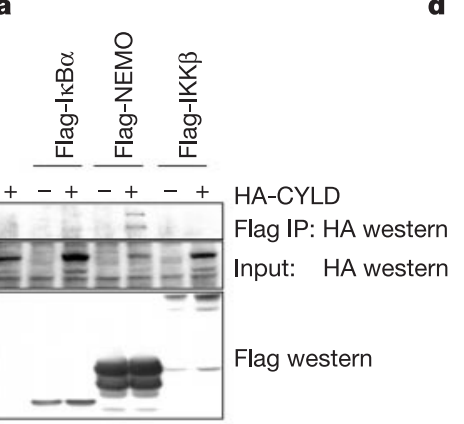

b

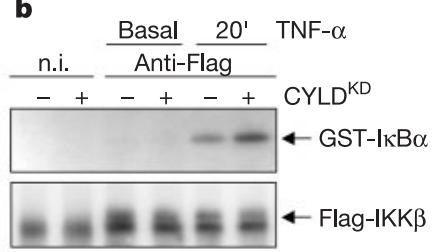

c

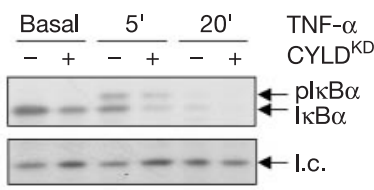

Figure 2 CYLD is an antagonist of NF- $\mathrm{kB}$ signalling. a, HEK293 cells were transfected as indicated, and immunoprecipitated (IP) using Flag antibody. IPs were immunoblotted for HA-tagged CYLD (upper panel), whole cell extracts were immunoblotted for Flag-tagged IкB $\alpha$, IKK $\beta$ and NEMO (lower panel) and for HA-tagged CYLD (middle panel). b, Flag-IKK $\beta$ was immunoprecipated with either non-immune serum (n.i.) or Flag antibody from both untreated cells (basal) or cells treated for 20 min. with TNF- $\alpha\left(20^{\prime}\right)$ and lysates were incubated with GST-IKB $\alpha$ (1-72) in the presence of ${ }^{32} \mathrm{P}-\gamma$ ATP (upper panel). Immunoprecipitated IKK $\beta$ was visualized by immunoblotting with Flag antibody (lower panel). c, U2-OS cells electroporated with pSUPER-CYLD or empty vector were stimulated with TNF- $\alpha$ and immunoblotted for $I_{k} B \alpha$ (I.c., loading control). d, U2-OS cells were transfected and immunoblotted for $\mathrm{IKB}_{\mathrm{K}}$. e, HEK293 cells were transfected and TRAF2 was immunoprecipitated using Flag antibodies. Immunoprecipitates were immunoblotted for HA-tagged ubiquitin (upper panel) and whole cell extracts for Flag-TRAF2 (lower panel).

CYLD knockdown could be reversed by the NF-кB inhibitor sodium salicylate. We tested this by treating HeLa cells with PMA in the presence or absence of pSUPER-CYLD. Apoptosis was induced by the addition of TNF- $\alpha$ and CHX. CYLD knockdown and PMA treatment again conferred resistance to TNF- $\alpha$-induced apoptosis (Fig. 4c). Exposure of cells to sodium salicylate abolished the protective effect of CYLD knockdown on apoptosis (Fig. 4c). This

a
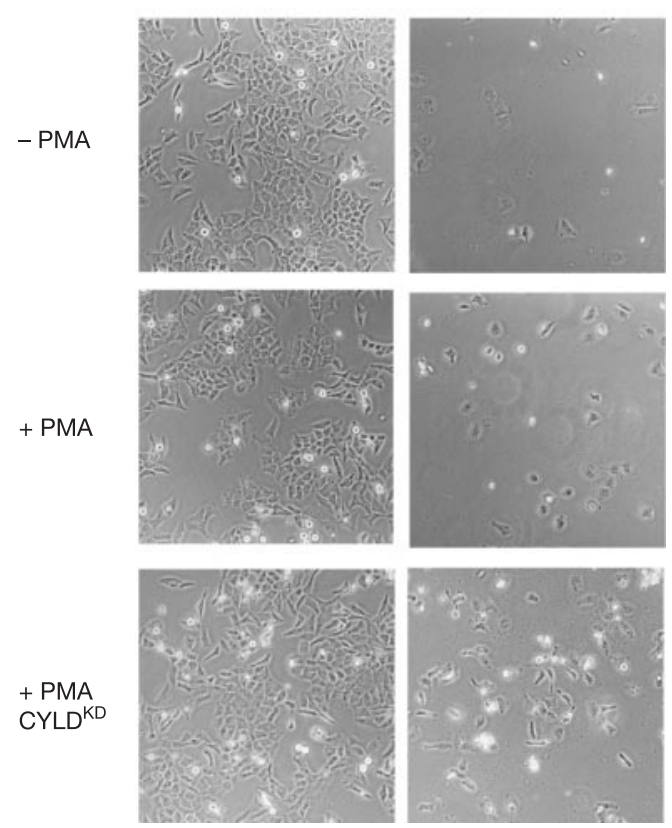

b

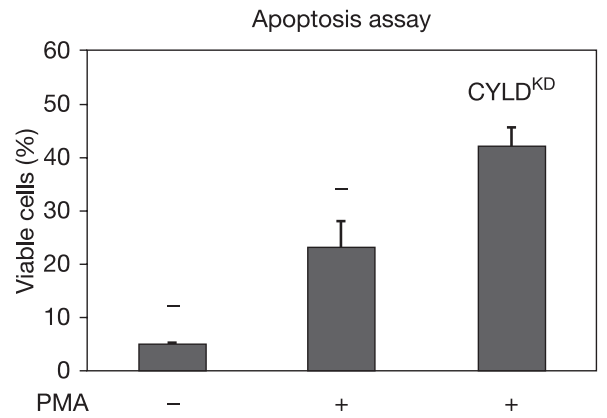

c

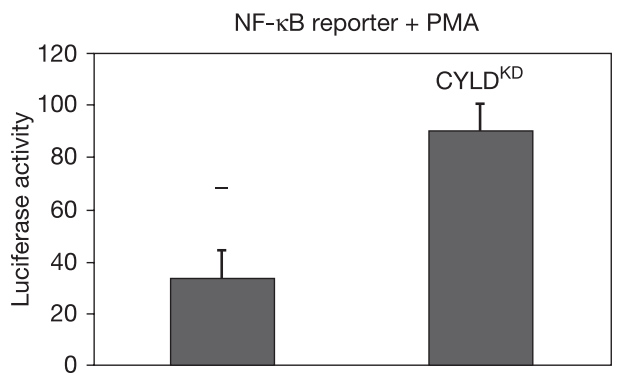

Figure $\mathbf{3}$ CYLD loss protects against TNF- $\alpha$ induced apoptosis. $\mathbf{a}, \mathbf{b}$, HeLa cells were electroporated as indicated. To stimulate NF-кB, cells were pre-treated with PMA for 2 hours or left untreated prior to inducing apoptosis by the simultaneous addition of TNF- $\alpha$ and $\mathrm{CHX}$. Photos were taken 16 hours after TNF- $\alpha / \mathrm{CHX}$ addition (a) and percentage of viable cells was quantified. The average of three independent experiments ( \pm s.d.) is shown (b). c, HeLa cells were transfected with a NF-KB luciferase reporter and PSUPER-CYLD or empty vector. Cells were stimulated with PMA for two hours and luciferase activity was measured. The average value of three independent transfections ( \pm s.d.) is shown. 

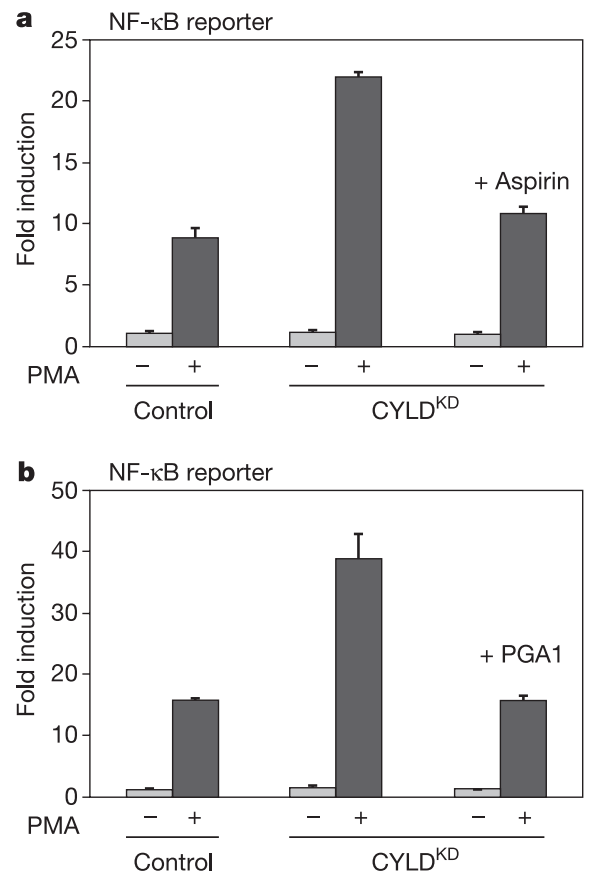

Figure 4 Anti-apoptotic effects of CYLD loss can be reversed by aspirin. $\mathbf{a}, \mathbf{b}$, U2-OS cells were transfected as in Fig. 1c, and either left untreated, stimulated with PMA, PMA and sodium salicylate (a, labelled 'aspirin') or PMA and prostaglandin A1 (b) and luciferase activity was measured. The average values of three independent transfections

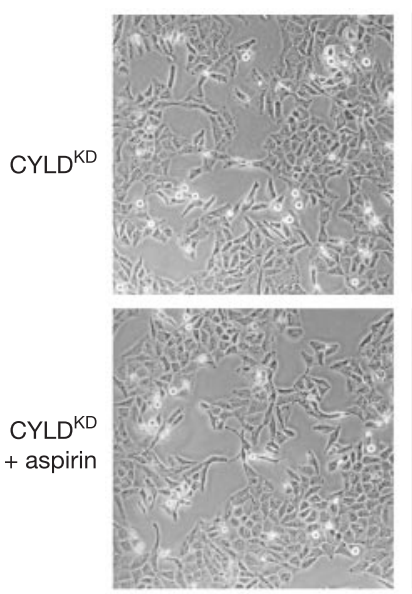

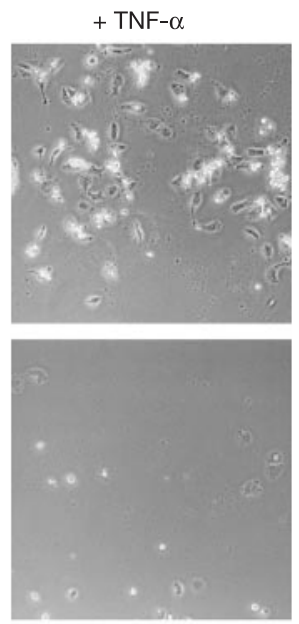

d

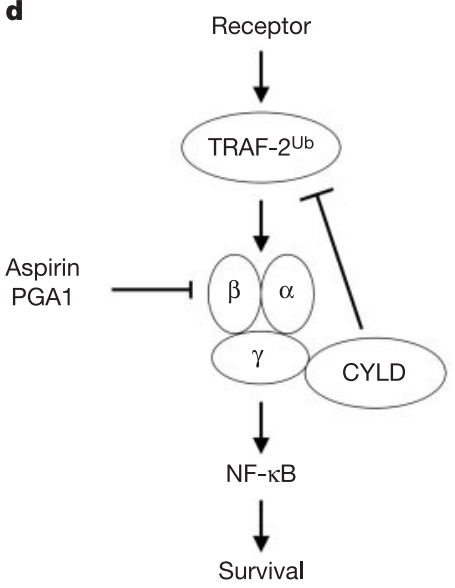

( \pm s.d.) are shown. c, Electroporated HeLa cells were pre-treated with PMA or PMA and sodium salicylate (labelled 'aspirin') for two hours. Apoptosis was induced by addition of TNF- $\alpha$ and CHX. Photos were taken 16 hours later. $\mathbf{d}$, Model of the consequences of CYLD loss on NF-kB activity and cell survival.

indicates that CYLD loss is anti-apoptotic through the activation of IKK $\beta$ and subsequently NF-кB.

We describe here a high-throughput RNA interference screen in mammalian cells to identify novel regulators of NF-кB. We focused on ubiquitin-specific processing proteases, as these proteins are antagonists of the ubiquitin conjugating enzymes and ubiquitin ligases. We identified the familial cylindromatosis tumour suppressor gene $(C Y L D)$ as a novel negative regulator of NF- $\mathrm{kB}$, thus establishing the first (to our knowledge) direct link between the NF-кB signalling cascade and a tumour suppressor gene. We propose a model in which CYLD functions to turn TNF-receptor signalling off by removing ubiquitin from TRAF2 (Fig. 4d). Although CYLD may have other targets than NF-кB, we suggest that the deregulated proliferation in the skin appendages in familial cylindromatosis results from a mild shift in the balance between proliferation and apoptosis in favour of proliferation. This is supported by the observation that most cylindromas have a diploid karyotype and are rarely metastatic ${ }^{4}$.

In the vast majority of cell types, activation of NF- $\mathrm{kB}$ is an oncogenic event ${ }^{9}$. However, it has recently been shown that inhibition of NF-кB in keratinocytes contributes to neoplasia ${ }^{22}$. Importantly, cylindromas are not derived from keratinocytes but from hair follicles and exocrine glands ${ }^{23}$. These skin appendages

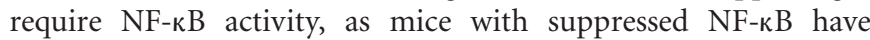
defects in the development of these tissues due to increased apoptosis $^{24,25}$.

Our observation that enhanced protection from apoptosis resulting from CYLD suppression can be reversed by simple pharmacological agents like sodium salicylate and prostaglandin A1 suggests a strategy to restore normal growth control in patients suffering from familial cylindromatosis. We are currently testing this in a clinical trial using topical application of aspirin derivatives on cylindromas.

\section{Methods}

\section{Materials, antibodies, and plasmid construction}

To generate DUB knockdown vectors, four annealed sets of oligonucleotides encoding short hairpin transcripts corresponding to one DUB enzyme (see Supplementary Information) were cloned individually into pSUPER. Bacterial colonies were pooled and used for plasmid preparation. To generate GFP-DUB fusion proteins, the corresponding DUB enzymes were PCR amplified and cloned into pEGFP-N1 (Clontech). pNF-кB-Luc vector was obtained from Clontech, SV40-Renilla from Promega. PMA, TNF- $\alpha$, prostaglandin Al and cycloheximide were purchased from Sigma. HA-tagged CYLD was PCR amplified, and cloned into pCDNA 3.1 (-), Flag tagged NEMO was generated by cloning an EcoRI-XbaI NEMO containing fragment into pcDNA-flag. Anti-IкB $\alpha$ (c-21) and HA tag (Y-11) antibodies were obtained from Santa Cruz, anti-Flag M2 from Sigma and anti-GFP rabbit polyclonal serum was provided by J. Neefjes.

\section{Cell cultures, transient transfections and reporter assays}

All cells were cultured in Dulbecco's modified Eagle medium (DMEM) supplemented with $10 \%$ fetal calf serum. High efficiency electroporation of cells was done as described ${ }^{26}$. Reporter assays were carried out using calcium phosphate transfection of $0.5 \mu \mathrm{g}$ NF-kBLuc, 1 ng SV40-Renilla and $2.5 \mu \mathrm{g}$ pSUPER vectors. Forty-eight hours after transfection, cells were stimulated with $200 \mathrm{nM}$ PMA or $20 \mathrm{ng} \mathrm{m}^{-1}$ TNF- $\alpha$ and luciferase activity was measured 72 hours after transfection. Sodium salicylate $(10 \mathrm{mM})$ or prostaglandin $\mathrm{Al}$ $(8 \mu \mathrm{M})$ was added to the cells 48 hours after transfection, and reporter activity was measured 72 hours after transfection. In HeLa cells, NF-kB activity was measured 2 hours after PMA stimulation.

\section{Immunoblotting, immunoprecipitation and kinase assay}

Western blots were performed using whole cell extracts, separated on 8-12\% SDS-PAGE gels and transferred to polyvinylidine difluoride membranes (Millipore). Western blots were probed with the indicated antibodies. HEK293 cells were transfected by calcium phosphate precipitation with the indicated plasmids; 48 hours after transfection cells were lysed in ELB buffer ( $0.25 \mathrm{M} \mathrm{NaCl}, 0.1 \%$ NP-40, $50 \mathrm{mM}$ HEPES pH 7.3) supplemented with 'Complete' protease inhibitors (Roche), and protein complexes were immunoprecipitated with $2 \mu \mathrm{g}$ of the indicated antibodies conjugated to protein $\mathrm{G}$ sepharose beads. To detect TRAF2 ubiquitination, immunoprecipitations were performed using RIPA buffer (50 mM Tris pH 8.0, $150 \mathrm{mM} \mathrm{NaCl}, 1 \% \mathrm{NP}-40,0.5 \%$ deoxycholic acid and $0.1 \%$ SDS). Immunoprecipitation/kinase assays were performed essentially as described ${ }^{27}$.

\section{Apoptosis assays}

Experimental design. In the experiments described in Figs 3 and 4, we first treat cells with 
PMA or PMA in the presence of CYLD knockdown for 2-3 hours. This allows NF-кB to become active and stimulate expression of the anti-apoptotic NF- $\mathrm{kB}$ target genes. Then we add TNF- $\alpha$ and CHX at the same time. The CHX is added because TNF- $\alpha$ has two effects it induces apoptosis in a transcription-independent fashion, and induces NF- $\mathrm{kB}$ to protect from apoptosis, which requires transcription. By adding CHX and TNF- $\alpha$ at the same time, the anti-apoptotic effects of TNF- $\alpha$ are blocked (by CHX), leaving only the apoptotic signalling pathway intact. This is why combined treatment with TNF- $\alpha$ and CHX is such a powerful inducer of apoptosis. Thus, in this experimental set-up the apoptotic effects of TNF- $\alpha$ can only be rescued by activation of NF- $\mathrm{KB}$ target genes prior to $\mathrm{CHX}$ treatment (that is, through the combined effects of PMA + CYLD knockdown).

Seventy-two hours after electroporation of HeLa cells with the indicated plasmids, cells were treated with $200 \mathrm{nM}$ PMA for 2-3 hours to stimulate NF-кB activity, followed by a 12-hour incubation in medium containing both $10 \mathrm{ng} \mathrm{ml}^{-1} \mathrm{TNF}-\alpha$ and $10 \mu \mathrm{g} \mathrm{ml}^{-1} \mathrm{CHX}$ to induce apoptosis. Viable cells were quantified using the Trypan blue exclusion method. To inhibit NF- $\mathrm{\kappa B}$ activity, medium was supplemented with $10 \mathrm{mM}$ sodium salicylate 3.5 hours before TNF- $\alpha$ addition.

Received 12 March; accepted 8 May 2003; doi:10.1038/nature01811.

1. Wilkinson, K. D. Ubiquitination and deubiquitination: Targeting of proteins for degradation by the proteasome. Semin. Cell Dev. Biol. 11, 141-148 (2000)

. Chung, C. H. \& Baek, S. H. Deubiquitinating enzymes: Their diversity and emerging roles. Biochem. Biophys. Res. Commun. 266, 633-640 (1999).

D’Andrea, A. \& Pellman, D. Deubiquitinating enzymes: A new class of biological regulators. Crit. Rev. Biochem. Mol. Biol. 33, 337-352 (1998).

4. Bignell, G. R. et al. Identification of the familial cylindromatosis tumour-suppressor gene. Nature Genet. 25, 160-165 (2000)

Yin, M. J., Yamamoto, Y. \& Gaynor, R. B. The anti-inflammatory agents aspirin and salicylate inhibit the activity of IkB kinase- $\beta$. Nature 396, 77-80 (1998).

6. Nakamura, T., Hillova, J., Mariage-Samson, R. \& Hill, M. Molecular cloning of a novel oncogene generated by DNA recombination during transfection. Oncogene Res. 2, 357-370 (1988).

Papa, F. R. \& Hochstrasser, M. The yeast $D O A 4$ gene encodes a deubiquitinating enzyme related to a product of the human tre-2 oncogene. Nature 366, 313-319 (1993).

8. Brummelkamp, T. R., Bernards, R. \& Agami, R. A system for stable expression of short interfering RNAs in mammalian cells. Science 296, 550-553 (2002).

9. Karin, M., Cao, Y., Greten, F. R. \& Li, Z. W. NF-kB in cancer: From innocent bystander to major culprit. Nature Rev. Cancer 2, 301-310 (2002).

10. Smahi, A. et al. The NF-kB signalling pathway in human diseases: From incontinentia pigmenti to ectodermal dysplasias and immune-deficiency syndromes. Hum. Mol. Genet. 11, 2371-2375 (2002)

11. Trompouki, E. et al. CYLD is a deubiquitinating enzyme that negatively regulates NF-kB activation by TNFR family members. Nature 424, 793-796 (2003).

12. Bradley, J. R. \& Pober, J. S. Tumor necrosis factor receptor-associated factors (TRAFs). Oncogene $\mathbf{2 0}$ 6482-6491 (2001).

13. Chung, J. Y., Park, Y. C., Ye, H. \& Wu, H. All TRAFs are not created equal: Common and distinct molecular mechanisms of TRAF-mediated signal transduction. J. Cell Sci. 115, 679-688 (2002).

14. Shi, C. S. \& Kehrl, J. H. TNF-induced GCKR and SAPK activation depends upon the E2/E3 complex Ubc13-UevlA/TRAF2. J. Biol. Chem. 18, 15429-15434 (2003).

15. Wang, C. et al. TAK1 is a ubiquitin-dependent kinase of MKK and IKK. Nature 412, 346-351 (2001)

16. Holtmann, H., Hahn, T. \& Wallach, D. Interrelated effects of tumor necrosis factor and interleukin 1 on cell viability. Immunobiology 177, 7-22 (1988).

17. Wang, C. Y., Mayo, M. W. \& Baldwin, A. S. Jr TNF- and cancer therapy-induced apoptosis: Potentiation by inhibition of NF-KB. Science 274, 784-787 (1996).

18. Liu, Z. G., Hsu, H., Goeddel, D. V. \& Karin, M. Dissection of TNF receptor 1 effector functions: JNK activation is not linked to apoptosis while NF-kB activation prevents cell death. Cell 87, 565-576 (1996).

19. De Smaele, E. et al. Induction of gadd $45 \beta$ by NF-kB downregulates pro-apoptotic JNK signalling. Nature 414, 308-313 (2001).

20. Baud, V. \& Karin, M. Signal transduction by tumor necrosis factor and its relatives. Trends Cell Biol. 11, 372-377 (2001)

21. Rossi, A. et al. Anti-inflammatory cyclopentenone prostaglandins are direct inhibitors of ІкB kinase Nature 403, 103-108 (2000).

22. Dajee, M. et al. NF-кB blockade and oncogenic Ras trigger invasive human epidermal neoplasia. Nature 421, 639-643 (2003)

23. van Balkom, I. D. \& Hennekam, R. C. Dermal eccrine cylindromatosis. J. Med. Genet. 31, 321-324 (1994).

24. Schmidt-Ullrich, R. et al. NF-kB activity in transgenic mice: Developmental regulation and tissue specificity. Development 122, 2117-2128 (1996).

25. Schmidt-Ullrich, R. et al. Requirement of NF-kB/Rel for the development of hair follicles and other epidermal appendices. Development 128, 3843-3853 (2001).

26. Agami, R. \& Bernards, R. Distinct initiation and maintenance mechanisms cooperate to induce G1 cell cycle arrest in response to DNA damage. Cell 102, 55-66 (2000).

27. Chen, G., Cao, P. \& Goeddel, D. V. TNF-induced recruitment and activation of the IKK complex require Cdc37 and Hsp90. Mol. Cell 9, 401-410 (2002).

Supplementary Information accompanies the paper on www.nature.com/nature

Acknowledgements We thank S. Lens, A. Lund and J. Borst for reagents, and M. Madiredjo for assistance. This work was supported by the Centre for Biomedical Genetics (CBG) and the Netherlands Organization for Scientific Research (NWO). A.D. was supported by a long-term fellowship from EMBO.

Competing interests statement The authors declare that they have no competing financial interests.

Correspondence and requests for materials should be addressed to R.B. (r.bernards@nki.nl).

\section{The tumour suppressor CYLD negatively regulates NF- $\kappa$ B signalling by deubiquitination}

\author{
Andrew Kovalenko ${ }^{1,2}$, Christine Chable-Bessia ${ }^{2}$, \\ Giuseppina Cantarella ${ }^{1,3}$, Alain Israël ${ }^{2}$, David Wallach ${ }^{1}$ \& Gilles Courtois ${ }^{2}$
}

${ }^{1}$ Department of Biological Chemistry, The Weizmann Institute of Science, 76100 Rehovot, Israel

${ }^{2}$ Unité de Biologie Moléculaire de l'Expression Génique, CNRS URA 2582, Institut Pasteur, 75015 Paris, France

${ }^{3}$ Department of Experimental and Clinical Pharmacology, University of Catania School of Medicine, I-95125 Catania, Italy

NF-кB transcription factors have key roles in inflammation, immune response, oncogenesis and protection against apopto$\operatorname{sis}^{1,2}$. In most cells, these factors are kept inactive in the cytoplasm through association with $\mathrm{I} \kappa \mathrm{B}$ inhibitors. After stimulation by various reagents, IкB is phosphorylated by the IкB kinase (IKK) complex ${ }^{3}$ and degraded by the proteasome, allowing NF-кB to translocate to the nucleus and activate its target genes. Here we report that CYLD, a tumour suppressor that is mutated in familial cylindromatosis ${ }^{4}$, interacts with NEMO, the regulatory subunit of IKK ${ }^{5,6}$. CYLD also interacts directly with tumournecrosis factor receptor (TNFR)-associated factor 2 (TRAF2), an adaptor molecule involved in signalling by members of the family of TNF/nerve growth factor receptors. CYLD has deubiquitinating activity that is directed towards non-K48-linked polyubiquitin chains, and negatively modulates TRAF-mediated activation of IKK, strengthening the notion that ubiquitination is involved in IKK activation by TRAFs and suggesting that CYLD functions in this process. Truncations of CYLD found in cylindromatosis result in reduced enzymatic activity, indicating a link between impaired deubiquitination of CYLD substrates and human pathophysiology.

The regulatory subunit of IKK, NEMO (also known as IKK- $\gamma$ ), has an essential role in NF- $\kappa \mathrm{B}$ activation and its dysfunction is associated with several distinct human pathologies ${ }^{7}$. On applying the carboxy-terminal half of NEMO as a bait in two-hybrid screening, we found that it bound specifically to the tumour suppressor CYLD (Fig. 1a). CYLD was originally identified through positional cloning of the gene responsible for familial cylindromatosis, an autosomal dominant disease characterized by benign tumours derived from cells of skin appendages, which appear predominantly in hairy areas of the body during adulthood ${ }^{4}$.

The binding site for CYLD on NEMO was found to correspond to the last 39 amino acids of the NEMO protein, where a zinc-finger is located (Fig. 1a). In further two-hybrid tests, CYLD was found to bind to TRAF2 but not to other members of the TRAF family or to any of the following signalling proteins: TRADD, RIP, RIP2, NIK, FADD, cFLIP, caspase 8, APAF1, IAP-1, the p55 TNFR intracellular domain, FAS-IC or A20 (data not shown). The whole TRAF domain of TRAF2 seemed to be required for the CYLD-TRAF2 interaction (Fig. 1b). We found that the NEMO-binding site is located between amino acids 470 and 684 in CYLD, whereas the TRAF2-binding site is located between CYLD amino acids 394 and 470. Because TRAF2 binds to the sequence motif Pro-X-Gln-X-(Ser/Thr $)^{8}$ and we found a similar sequence in the TRAF2-binding domain of CYLD (Pro 453-Val 454-Gln 455-Glu 456-Ser 457), we examined the participation of this sequence in the TRAF2-CYLD interaction by mutating Ser 457 to alanine. This mutation abolished TRAF2 binding to CYLD (Fig. 1c).

CYLD binding to NEMO and to TRAF2 could be also detected in transfected human embryonic kidney (HEK) 293T cells (Fig. 2a, d). 